\title{
CYTOLOGICAL EVALUATION OF METASTATIC MELANOMA- A SERIES OF 3 CASES
}

\author{
Sant Prakash'1, Ayushi Saxena², Sanjay Kumar³, Nilay Nishith4, Vartika Goel5, Rajeev Sen ${ }^{6}$
}

1Professor, Department of Pathology, Pandit B. D. Sharma, PGIMS, Rohtak, Haryana, India.

2Junior Resident, Department of Pathology, Pandit B. D. Sharma, PGIMS, Rohtak, Haryana, India.

3 Professor, Department of Pathology, Pandit B. D. Sharma, PGIMS, Rohtak, Haryana, India.

${ }^{4}$ Senior Resident, Department of Pathology, Pandit B. D. Sharma, PGIMS, Rohtak, Haryana, India.

5 Junior Resident, Department of Pathology, Pandit B. D. Sharma, PGIMS, Rohtak, Haryana, India.

${ }^{6}$ Senior Professor and HOD, Department of Pathology, Pandit B. D. Sharma, PGIMS, Rohtak, Haryana, India.

\section{ABSTRACT}

\section{BACKGROUND}

Malignant melanoma is an aggressive tumour with a propensity to metastasize. We report a series of 3 cases, which presented with lymphadenopathy and cytological diagnosis of metastatic melanoma was made based on morphology and immunocyto-chemical features. FNAC is a highly accurate, rapid, and cost-effective procedure for the diagnosis of metastatic melanoma and should be considered as the initial diagnostic procedure of choice in patients with melanoma with clinically suspected metastases.

\section{KEY WORDS}

Fine Needle Aspiration Cytology (FNAC), Lymph Node, Metastatic Melanoma.

HOW TO CITE THIS ARTICLE: Prakash S, Saxena A, Kumar S, et al. Cytological evaluation of metastatic melanoma- a series of 3 cases. J. Evolution Med. Dent. Sci. 2019;8(01):88-90, DOI: 10.14260/jemds/2019/19

\section{BACKGROUND}

Melanoma is an aggressive tumour, accounting for approximately $3 \%$ of all malignancies worldwide. ${ }^{1}$ However, it is relatively uncommon among the Indian population, with an incidence of $<0.5 \% .^{2}$ Melanomas have a high propensity to metastasize, usually to the draining lymph nodes. Significant mortality has been noted in patients with metastatic disease, thereby underscoring the importance of early detection of both primary and metastatic melanoma. ${ }^{3}$ In this regard, fine needle aspiration cytology (FNAC) of the lesion offers quick and accurate results. Herein, we report a series of three cases in which cytological diagnosis of metastatic melanoma to lymph node was made prior to histopathological assessment.

\section{CASE SERIES}

\section{Case 1}

35-year old male presented with swelling in the right loin since 4 months. Earlier, he had been operated for melanoma of right foot. On physical examination, a firm and fixed swelling measuring $3 \times 2 \times 1 \mathrm{~cm}$ was noted in the right inguinal region.

\section{Case 2}

An elderly female came with swelling in the right loin for past 1 month. On further probing, she unveiled the history of vulval melanoma, which was surgically excised. Physical examination revealed a firm, tender and fixed swelling measuring $1.5 \times 1 \times 1 \mathrm{~cm}$ in the right inguinal region.

'Financial or Other Competing Interest': None.

Submission 26-11-2018, Peer Review 21-12-2018,

Acceptance 29-12-2018, Published 07-01-2019.

Corresponding Author:

Dr. Ayushi Saxena,

\#105, Block A,

Gaur Ganga Apartments,

Vaishali, Ghaziabad,

Uttar Pradesh, India.

E-mail: ayushisxn01@gmail.com

DOI: $10.14260 /$ jemds $/ 2019 / 19$

\section{Case 3}

37-year old male presented with an axillary swelling since 5 months and no other complaints. Physical examination showed a swelling, firm in consistency, fixed to the underlying skin and measuring $2 \times 1 \times 0.5 \mathrm{~cm}$. On subsequent follow-up, the patient complained of ulceration in the right thumb, which was later diagnosed as melanoma on histopathology.

For all the three cases, the initial physical examination was followed up by routine haematological and biochemical investigations, which were within normal limits. The patients later underwent fine needle aspiration cytology (FNAC) using a 23-gauge needle. In the first case, the cytological procedure yielded a blackish material admixed with blood. Subsequently, cytomorphological analysis by light microscopy was performed. Leishman stained smears revealed sheets, clusters and singly scattered pleomorphic tumour cells with moderate amount of basophilic cytoplasm, round to oval hyperchromatic nucleus and few showing prominent nucleoli; along with bi-nucleated forms of tumour cells in a background of numerous lymphocytes and haemorrhage. (Figure 1) Characteristically the malignant cells also possessed intra-cytoplasmic fine, granular, blackishbrown pigments; which were morphologically suggestive of melanin. Based on the clinical and cytological findings, a provisional diagnosis of melanoma metastasizing to lymph node was made. Consequently, special stain i.e. FontanaMasson was performed, which revealed black coloured fine granules in the cytoplasm of tumour cells. (Figure 2) Furthermore, cell block was prepared from the aspirated material for ancillary study. Immunocytochemical evaluation with HMB-45 and S-100 resulted in positive staining pattern. Thus, confirming the diagnosis of metastatic melanoma to the lymph node. (Figure 3) The other two cases also displayed similar cytomorphological and immunocytochemical features and hence, they were also labelled with the same diagnosis. Subsequently, all the three patients underwent surgical excision of the lesion and are currently on regular follow-up in the department of surgery. None of the cases have demonstrated any sign of recurrence. 

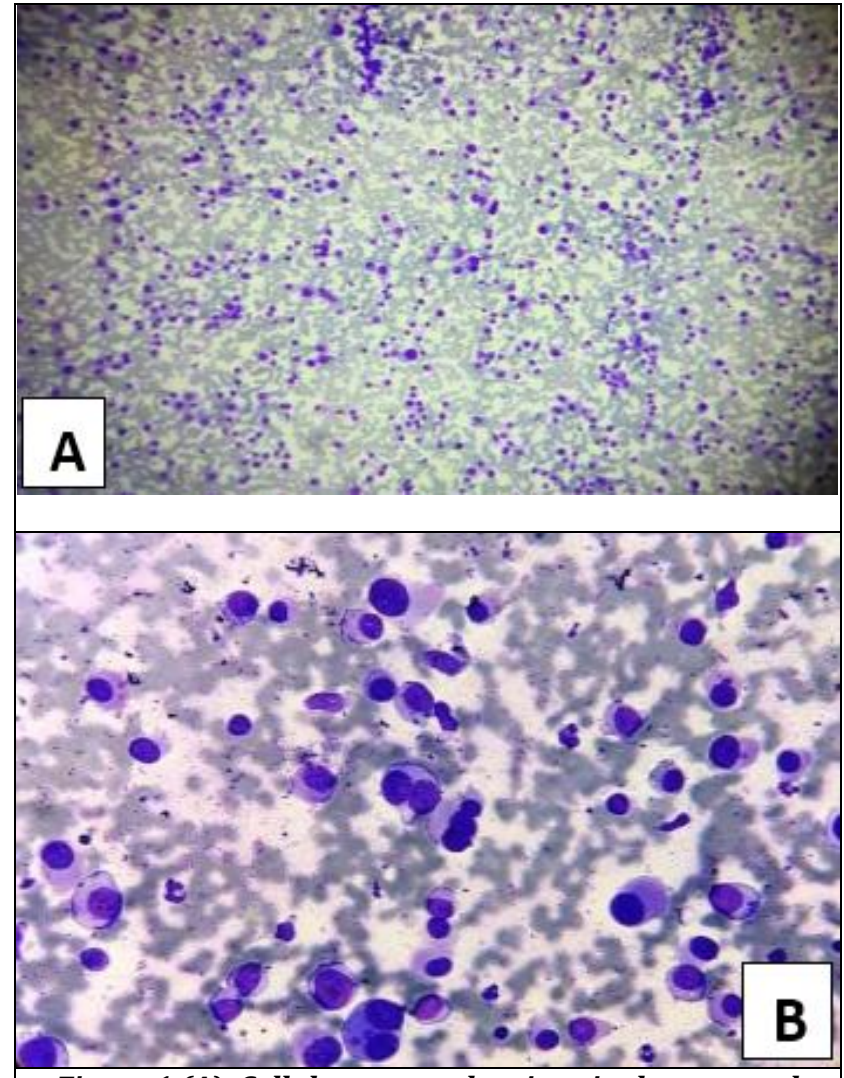

Figure 1 (A). Cellular smear showing singly scattered pleomorphic tumour cells (Leishman stain; X100). (B) Cells have moderate amount of basophilic cytoplasm with eccentrically located, round to oval hyperchromatic nucleus (Leishman stain; X400)

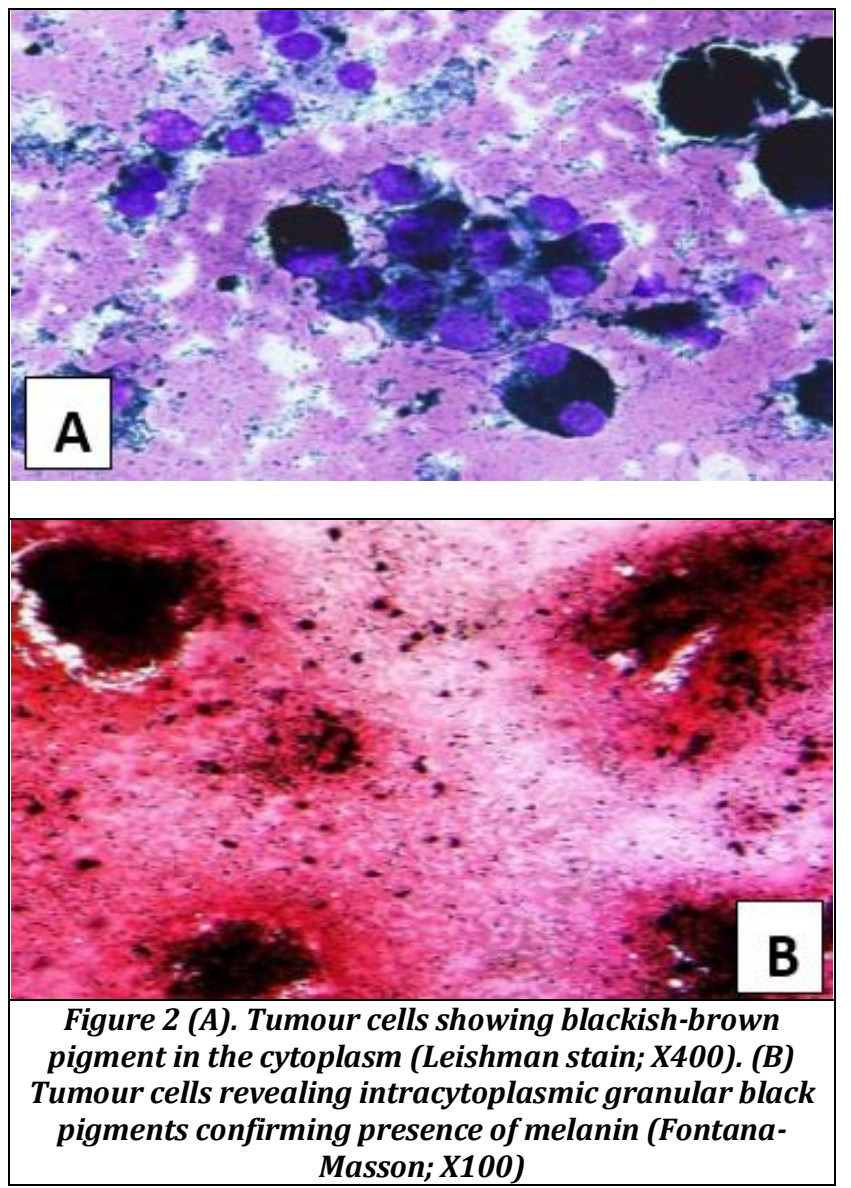

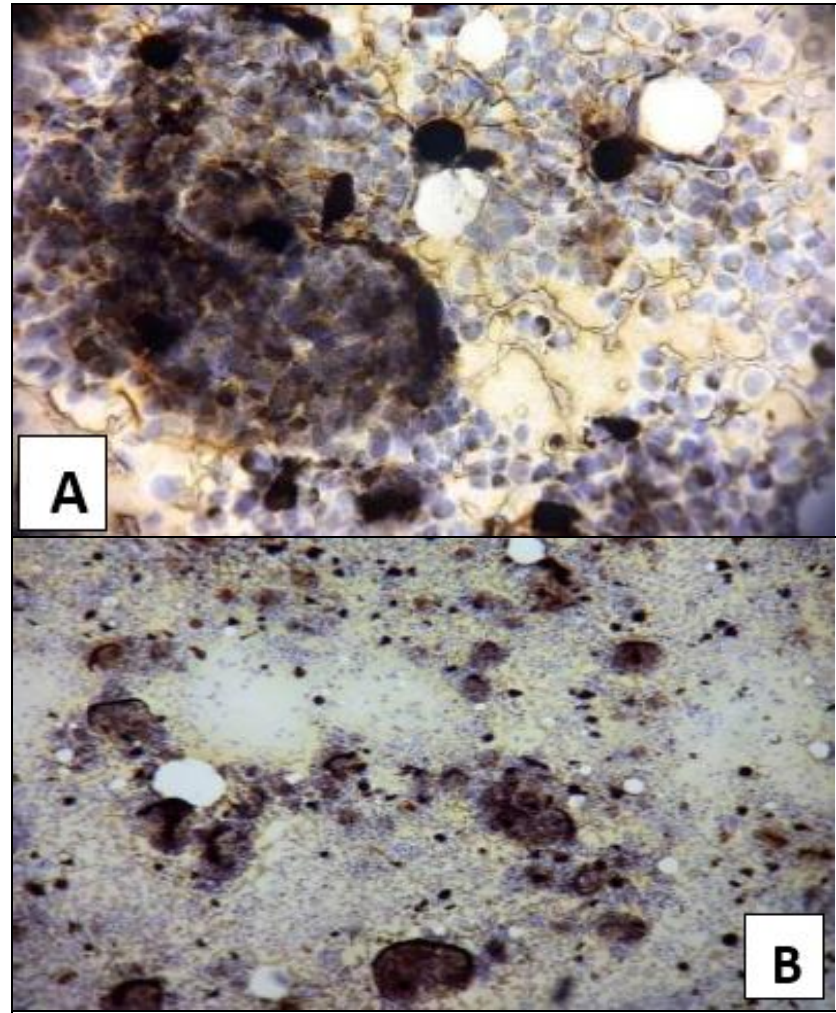

Figure 3. Tumour cells demonstrating immune-reactivity for (A) S-100 (ICC; X400) (B) HMB-45 (ICC; X400)

\section{DISCUSSION}

Melanoma is an aggressive cutaneous malignancy that usually affects adolescent and middle-aged individuals. With regard to gender predisposition, young females and males over the age of 55 years, frequently present with melanoma. Ultraviolet radiation from sunlight is the key risk factor for cutaneous melanoma. Apart from environmental factors, genetic mutations and alterations in the tumour microenvironment also contribute to the pathogenesis of melanoma. Genomic dysregulation in BRAF, NRAS, NF1, and KIT genes are regarded as driver mutations for progression and metastasis of melanoma. ${ }^{4}$ Metastasis to the regional lymph node is commonly associated with melanoma; nonetheless, the exact mechanism for such a predilection remains unclear. Zybtek et al. postulated that few melanomas express chemokine or chemokine receptors and adhesion molecules that lead to preferential homing of tumour cells to lymph nodes. ${ }^{5}$

Amongst others, lymph node metastasis is the foremost predictor of survival in patients with melanoma. Therefore, an accurate and prompt assessment of lymph node status would expedite the therapeutic management and improve outcome. 6 In this regard, FNAC of the lesion offers rapid and reliable result when performed by a skilled hand. In addition, it is also cost-effective, safe and well-tolerated by patients. ${ }^{3}$

Melanomas are renowned for their capricious cytomorphology and ability to simulate almost any malignancy, thereby making it a diagnostic challenge. A literature review by Lindsey et al. details the characteristic cytological findings that were seen in an aspirate of metastatic melanoma to lymph node; which include hypercellular smears composed of tumour cells that are either singly scattered or form three-dimensional clusters with indistinct cell borders. The cells are predominantly 
polygonal in shape and may be admixed with epithelioid or spindle-shaped cells. Polygonal cells have moderate to abundant amount of granular or clear cytoplasm with multiple small intracytoplasmic vacuoles. The nuclear features observed are eccentric nucleus, dense and dispersed chromatin, prominent macronucleoli, and intranuclear inclusion along with frequent multinucleation. Moreover, presence of melanin pigment in the tumour cells is the only cytomorphological feature to confidently diagnose melanoma. The pigment might be dusty or finely granular and is distributed evenly throughout the cytoplasm. ${ }^{7}$ Additionally, special stain i.e. Fontana-Masson and immunocytochemistry (ICC) with S-100 and HMB-45, are used to confirm metastatic melanoma. ${ }^{8}$

Several studies suggest pigment-laden macrophages as the main culprit for misleading a cytopathologist while evaluating metastatic melanoma to the lymph node. These cells often occur in various neoplastic and non-neoplastic conditions, thus causing a diagnostic dilemma for the cytopathologist. Tattoo lymphadenitis and dermatopathic lymphadenopathy (DLN) are the two common benign entities that can often mimic metastatic melanoma. Cytological examination of the former reveals histiocytes with intracytoplasmic carbon deposits, which are negative for HMB-45 and S-100 and the hallmark cytomorphological findings of the later, include sheets of histiocytes intervened by lymphoid cells and enclosed by numerous blood vessels. These cells have abundant, wispy cytoplasm with indistinct cell border, elongated or round to oval vesicular nucleus with fine nuclear chromatin, nuclear grooving, and absent or inconspicuous nucleoli. Also, melanin-laden histiocytes are common in DLN, which show positivity for S-100 and CD68. On the other hand, pigmented basal cell carcinoma, a cutaneous malignant tumour, can also simulate melanoma when it metastasizes to the lymph node. Additionally, melanoma being a great mimicker, can exhibit pleomorphic morphology and emulate various epithelial and nonepithelial malignancies; which could further cloud the judgment of a cytopathologist. ${ }^{8},{ }^{9}$ Hence, it is prudent to conduct a thorough clinical and cytomorphological analysis for precisely diagnosing metastatic melanoma. In challenging situations, Fontana-Masson special stain and ICC definitely aid in reaching a conclusive decision. In our case series, based on the history and cytopathologic features, a provisional diagnosis of metastatic melanoma to lymph node was made. We also employed special stain and ICC, in order to validate our diagnosis and exclude the probable differential diagnoses. In addition, a paucity of cytological studies on metastatic melanoma in India, made it pertinent for us to report this case series.

In the past few years, we have embarked a milestone in the management of both primary and metastatic melanoma. There are numerous therapeutic modalities for treating melanoma such as surgical resection, chemotherapy, electrochemotherapy, bio-chemotherapy, radiotherapy, photodynamic therapy (PDT), immunotherapy, and targeted therapy. However, the metastatic disease is essentially dealt with surgical resection and chemotherapy. Recent developments have emphasized the role of immunotherapeutic agents (Interleukin-2) in managing patients with metastatic disease. Nivolumab is the latest and most-efficient FDA approved immunomodulatory drug indicated for the treatment of metastatic melanoma. ${ }^{10}$

\section{CONCLUSION}

Melanoma is an aggressive tumour with staggering metastatic potential and a morbid outcome. In this regard, a detailed clinical history and cytological evaluation of the lesion can offer an early and accurate diagnosis. Nonetheless, a cytopathologist faces several challenges while diagnosing metastatic melanoma in a lymph node. Therefore, we advocate a meticulous assessment of the neoplasm by the surgeon and to consider FNAC before resorting to biopsy.

\section{REFERENCES}

[1] Murali R, Doubrovsky A, Watson GF, et al. Diagnosis of metastatic melanoma by fine-needle biopsy: analysis of 2, 204 cases. Am J Clin Pathol 2007;127(3):385-97.

[2] Boyle P, Maisonneve P, Dore JF. Epidemiology of malignant melanoma. Br Med Bull 1995;51(3):523-47.

[3] Rodrigues LK, Leong SP, Ljung BM, et al. Fine needle aspiration in the diagnosis of metastatic melanoma. J Am Acad Dermatol 2000;42(5 Pt 1):735-40.

[4] Leonardi GC, Falzone L, Salemi R, et al. Cutaneous melanoma: from pathogenesis to therapy (Review). Int J Oncol 2018;52(4):1071-80.

[5] Zbytek B, Carlson JA, Granese J. Current concepts of metastasis in melanoma. Expert Review of Dermatol 2008;3(5):569-85.

[6] Balch CM, Gershenwald JE, Soong SJ, et al. Final version of 2009 AJCC melanoma staging and classification. J Clin Oncol 2009;27(36):6199-206.

[7] Lindsey KG, Ingram C, Bergeron J, et al. Cytological diagnosis of metastatic malignant melanoma by fineneedle aspiration biopsy. Seminars in Diagnostic Pathol 2016;33(4):198-203.

[8] Siddaraju N. Clinical cytology in the diagnosis and management of melanoma. Jawaharlal Institute of Postgraduate Medical Education and Research (JIPMER) India, September 22nd 2011.

[9] Iyer VK, Kapila K, Verma K. Fine needle aspiration cytology of dermatopathic lymphadenitis. Acta Cytologica 1998;42(6):1347-51.

[10] Domingues B, Lopes JM, Soares P, et al. Melanoma treatment in review. Immunotargets and Therapy 2018;7:35-49. 